\title{
La obra de Medardo Rosso como punto de encuentro entre una estética clásica y otra contemporánea
}

\author{
Guillermo Aguirre MarTínEZ \\ Universidad Complutense de Madrid. \\ Teoría de Literatura y Literatura Comparada \\ guillermo-aguirre@hotmail.com
}

Recibido: 15-01-2014

Aceptado: 28-10-2014

\section{RESUMEN}

La obra de Medardo Rosso (1858-1928) se configura, junto con la de Degas y Rodin, como iniciadora de la escultura moderna en la medida en que en ella se sustituyen una serie de motivos heredados de la estética tradicional, por conceptos hasta entonces apenas desarrollados. De este modo, según veremos, rasgos tendentes a la abstracción y a la desmaterialización se impondrán de manera progresiva otorgando un tinte impresionista, fugaz, a las realizaciones del artista italiano.

Por otra parte, observaremos ciertos modelos escultóricos precedentes sobre los que afianzar la obra de Rosso, con el propósito de acercarnos con la perspectiva adecuada hacia una serie de innovaciones llamadas a pervivir en el desarrollo de la plástica contemporánea.

Palabras clave: Medardo Rosso, escultura, transi, Impresionismo.

\section{Medardo Rosso's Work as a Meeting point between Classical and Contemporary Aesthetics}

\begin{abstract}
Medardo Rosso, with Degas and Rodin, is considered the initiator of modern sculpture due to giving up the traditional influences and also due to his attempt to introduce unknowed or unnoticed aspects until that moment. As we will see, new characteristics will be developed in order to conquer a high level of abstraction and to achieve the dematerializatio of the object. An impressionistic hue, a fleeting aspect, will be considered as a common feature of Rossos's sculpture.

Moreover, we will notice the approach between Rosso's work and another predecessor models with the purpose of following the trail of certain aspects that runs from the past to the contemporary sculpture.
\end{abstract}

Key words: Medardo Rosso, sculpture, transi, Impresionism. 


\section{Medardo Rosso como iniciador de la escultura moderna}

La obra de Medardo Rosso puede comprenderse como el advenimiento definitivo de una estética entregada más a la presentación de lo difuso que a la manifestación de lo apolíneo y plenamente delimitado. Como iniciador, junto a Rodin y Degas, de una escultura impresionista -si bien es cierto que en el caso de este último sus obras quedaron desconocidas a lo largo de su vida-, Rosso resaltará de la materia no su superficie sino su dinamismo interno, no su cristalización definitiva sino el proceso larvario que conduce a una coagulación comprendida desde la corriente impresionista como percepción falsa y por ello antiestética de la propia vida. De este modo, la materia, presagiando ya una percepción de la naturaleza defendida desde cierto sector de las futuras vanguardias -tal y como podremos observar en el arte surrealista o en la escultura biomórfica-, participará de la constante necesidad de presentar sus particulares procesos de metamorfosis, dejando de lado una estratificación inerte e irreal. La obra de arte, de acuerdo con lo indicado, se comprenderá como naturaleza, y ésta como continua renovación, reacia a mostrarse desde una hierática quietud enfrentada a un devenir existencial inexorable.

En relación con esta primacía de la organicidad propia de la obra de Rosso, resulta destacable que "he is at the opposite pole from Apollonian finitude, Pythagorean geometrics, Platonism or its academic relative, of explanatory, apologetic or ruling design. His sculptures are inmersed in a hollowed-out world, devoid of any consistency, weight or rigour -the realm of Dionysius. Subterranean even". ${ }^{1}$ Según observamos, como partícipe de una estética finisecular orientada a derribar el orden propio de la representación apolínea, Rosso comprenderá el hecho estético como fenómeno a través del cual resulta posible la presentación artística de un bullente interior dionisíaco y dinámico, enemigo de toda visión esclerotizada de la realidad y, en consecuencia, más cercano a un universo pagano y sensible que a una comprensión de cuño monoteísta. La materia cobrará vida por sí misma y por sí misma se deformará hasta su transformación en nueva configuración de elementos. En este sentido, cabe indicar, la representación clásica de naturaleza dual será sustituida por un modelo donde materia y dinámica espiritual -si es que resulta posible hablar de esta última en la obra de Rosso- se confunden en un solo cuerpo para así abrir paso a un desarrollo y decaimiento conjunto. ${ }^{2}$ Paralelamente a este proceso, destaca el desarrollo durante este periodo finisecular de un cúmulo de doctrinas de raíz órfica -teosofía y antroposofía-, que se irán abriendo paso en la estética advenediza dejando por aquí y por allá una serie de tratados ocultistas ${ }^{3}$ llamados a irradiar, con su nueva concepción de la naturaleza y el

1 MOLA, Paola "The Transient Form”, en MOLA, Paola. (ed). Rosso. The Transient Form. Milán: Skira, 2007, 17-27, p. 20.

2 Resulta destacable al respecto el hecho de que es en este periodo de comienzos del siglo XX cuando Ernst Rutherford desarrollará su teoría en torno a la automoción de la materia, hallazgo clave para comprender la visión de la naturaleza por parte del individuo moderno.

3 La primera edición de La doctrina secreta, de Helea Blavatsky, data de 1888. El libro ejercerá desde el primer momento una influencia considerable en diferentes artistas del momento, quienes trasladarán las ideas expuestas a su obra, caso de Mondrian o de Kandinsky. 
orden trascendental, una creación entendida desde la traslación de lo apolíneo solar al flujo constante de lo permanentemente renovado.

Como señalábamos, de modo paralelo a esta introspección en la naturaleza camina el deseo de superación de un atavismo en el que la representación del individuo había quedado encorsetada desajustándose así el libre equilibrio requerido para la articulación de lo propiamente racional dentro de un sistema de factores dinámicos y no artificiales. La escultura de Rosso, por consiguiente, dado su espíritu rupturista, quedó llamada a abrir una brecha en el mundo de la representación para permitir el surgimiento incoercible del espíritu -comprendido ahora como mera dinámica material-, concediendo al hecho artístico una cualidad curativa tan del gusto de las corrientes herméticas aludidas. La obra de arte, de esta manera, se presentaba como elemento de proyección de aquellas energías caóticas enclaustradas en el seno del individuo posibilitando la liberación de un intenso torrente vital abierto hacia un universo de formas en permanente expresión, en infinita y rica multiplicidad.

La labor del escultor italiano se entregará así a destacar la fugacidad del momento, decantándose por plasmar la realidad desde un exclusivo acercamiento que denotará, al deslizar nuestra mirada sobre la superficie, un momento perdido e irrecuperable, devolviéndonos una experiencia similar a la que por aquellas mismas fechas se esforzaba en describir Marcel Proust en el curso de su obra. ${ }^{4}$ La unidireccionalidad de la obra quedará de esta manera rota requiriendo de nuestra actividad si es se quiere dar con ese enfoque exacto desde el que, quizás, un hallazgo nos logre sorprender. Esta necesidad de descubrir la perspectiva adecuada se presentará como búsqueda y encuentro de lo atemporal dentro de lo sometido al devenir histórico. Se alcanza con ello un rapto de contemplación, una inmersión del espectador en el universo pleno de la obra, un cosmos donde las leyes y los sistemas de medida usuales quedan abolidos y sustituidos por el hallazgo de lo eterno y permanente. La realización, poseedora de su propia temporalidad, solapará en su cuerpo un orden externo y uno interno, quedando así como ámbito de reunión o de separación, como límite, en fin, de una dualidad de espacios en ese momento abolidos. ${ }^{5}$

En este mismo sentido, la característica veladura con que se cubren las figuras realizadas por Rosso apartará al objeto representado del orden de lo temporal, dado que dicho objeto quedará como embalsamado, envuelto en una crisálida donde se

\footnotetext{
4 Reveladoras se muestran las palabras de Marcel Proust recogidas en su obra Contra Sainte-Beuve: "Y de esa impresión, y de todas las semejantes, se desprende algo que les es común, algo cuya superioridad a las realidades de nuestra vida, incluso a las de la pasión, la inteligencia y el sentimiento, nos vemos capaces de explicar. Pero esa superioridad es tan patente que viene a ser lo único de lo que no podemos dudar. En el momento en que ese algo, esencia común de nuestras impresiones, es percibido por nosotros, experimentamos un placer sin parangón, durante el cual sabemos que la muerte es algo sin importancia" Marcel, PROUST, Contra Sainte-Beuve. Recuerdos de una mañana, Barcelona, Tusquets, 2005, p. 88).

5 En este sentido, Paola Mola sentencia que "Rosso plunges into sensations but denies any possibility of transforming the apprehension of the senses into something permanent. This is perhaps the aspect of the famous unique viewpoint in which the work of Rosso gained the significance that we feel is closest to us. One sees his work, and in moving forwards no longer sees it. It was the only way to preserve movement in sculpture. [...] The image appeared and disappeared, touchingly and unsuspectingly in the casual position of lights and shadows in between the folds of wax or bronze magma. Once again, Light formed another reality" (MOLA, Paola op. cit., p. 24).
} 
conservará la forma más o menos intacta de aquello que en su momento respiraba. Este recurso, qué duda cabe, denotará a su vez lo perecedero y mortal. El rostro esculpido, pese a conservar aún rasgos propios, comenzará a ser absorbido por la materia, quedando así como modelo proyectado no ya desde nuestro mundo apolíneo sino desde su hundimiento en la naturaleza. El presente empleo del velo o la crisálida que amortaja a las figuras, en consecuencia, llegará acompañado de una pérdida de individualidad. La materia, en estos momentos, comenzará a observarse carcomida, los ojos envueltos en una neblina como signo de su lento apagarse, de su innecesidad ya de permanecer abiertos. El modelo empezará a mostrar su superficie picada, presta a participar de una descomposición ni tan siquiera evitable por el cincel del artista, guardián de la esfera de lo eterno.

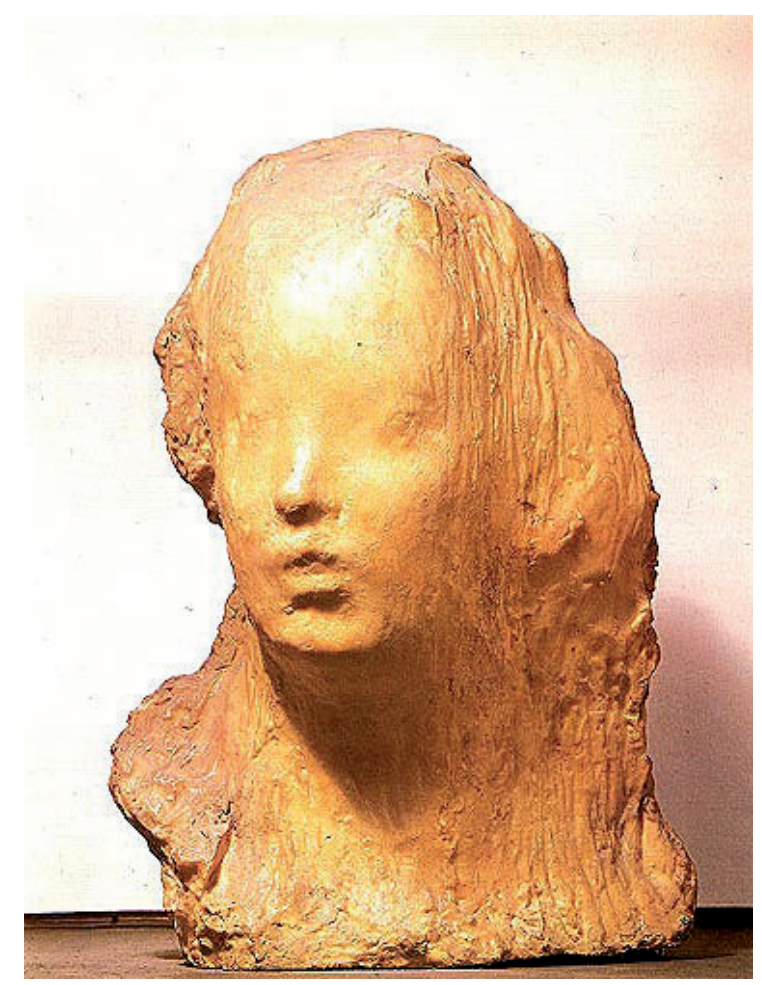

Fig. 1. Medardo Rosso, Ecce Puer, 1906. Galleria Ricci Oddi, Piacenza.

Como vemos, poco tendrá que ver esta propuesta escultórica con la estética dominante hasta poco tiempo atrás, donde el cuerpo, de aparecer cubierto por sudarios o velos, quedaba como protegido de lo perecedero, manifestándose en todo caso la caducidad de la materia junto a la pervivencia absoluta del espíritu. Valga señalar, como exposición de un consagrado triunfo del alma y del cuerpo, por ejemplo, el Cristo Velato de Giuseppe Sanmartino, donde tras el consignado velo se muestra a un Cristo incorruptible e inmortal, poseedor de una belleza ajena al deterioro de la materia. 


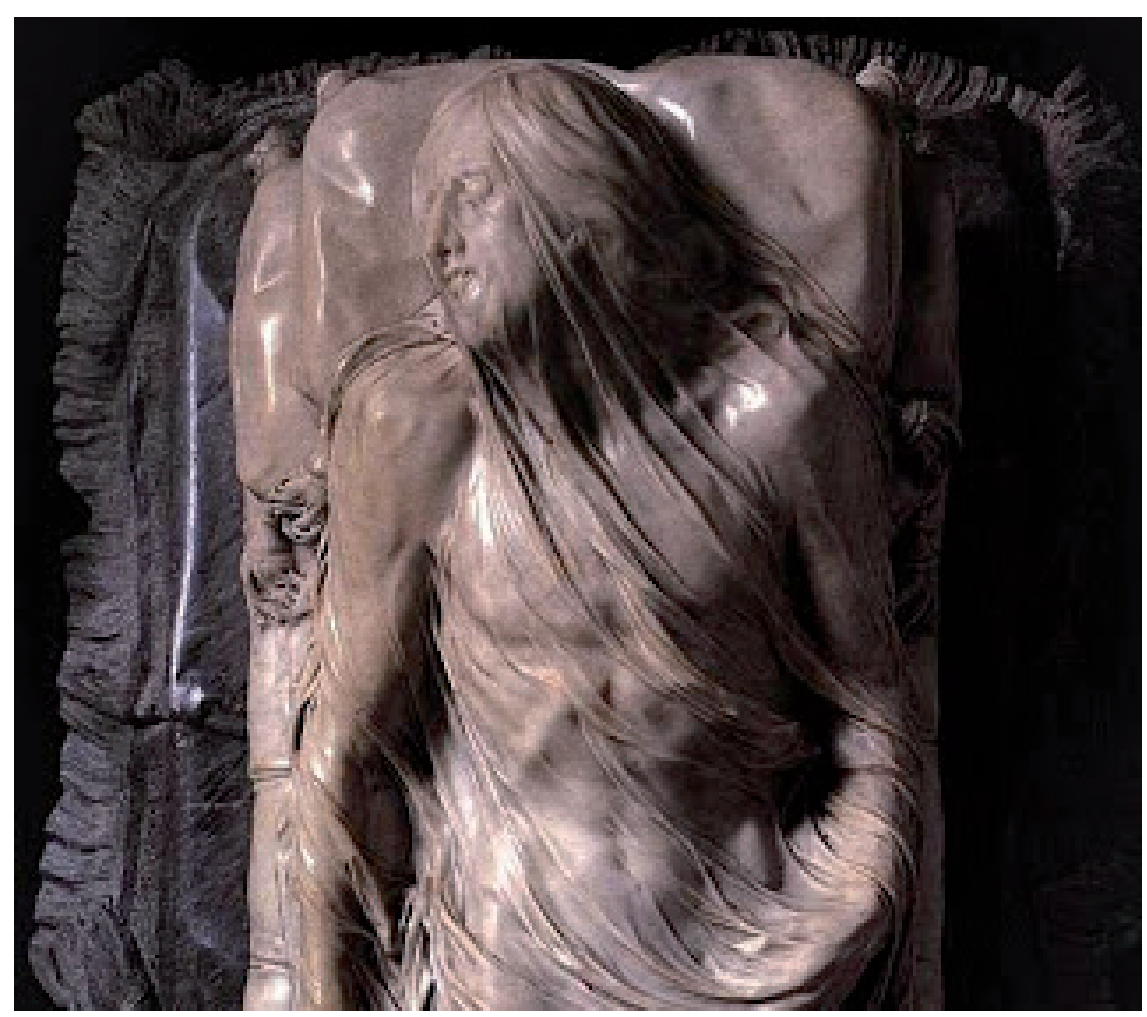

Fig. 2. Giuseppe Sanmartino, Il Cristo Velato, 1753. Museo Cappella Sansevero, Napoli.

Desde esta contraposición resulta perfectamente claro el modo en que se comprenderá, por un lado, la pervivencia de la corporeidad como hecho naturalmente aceptado en época de Sanmartino -siglo XVIII- y, por el otro, cómo ya en la época finisecular de Medardo Rosso esta misma corporeidad se observará entregada a la descomposición natural. Partiendo de esta dualidad parece posible establecer una asimilación entre la obra de Rosso y la larga tradición de transi observable en la escultura europea desde el siglo XIV hasta el XVII, dado que en ambos casos quedará patente ya un rechazo ya un escepticismo en lo relativo a la naturaleza divina del cuerpo, si bien es cierto que confiada en la eternidad del espíritu en el caso de los transi pero no así en la obra de Rosso. Desde este planteamiento podemos ver cómo dentro del ámbito de radiación de la Reforma, cuyos primeros síntomas los podemos encontrar en un cambio de mentalidad religiosa producido en fecha tan temprana como a inicios del siglo XIII, quedará patente el triunfo de la vida espiritual sobre la muerte, sobre aquella corporeidad que, desde un realismo exagerado, sería representada con tintes macabros ya en el coletazo final de la Edad Media. Habría que esperar aún unos pocos siglos para que pudiésemos contemplar a través de la obra de arte cómo la descomposición material caminaría de la mano de un deterioro espiritual tan 
sintomático como deudor del largo proceso dialéctico sufrido por occidente tendente a la reducción definitiva de las fuerzas trascendentales a lo meramente orgánico.

A continuación nos acercaremos a observar brevemente este desarrollo a través de los transi con el propósito de establecer una asimilación a nuestro juicio interesante desde la que podremos prestar especial atención a dos orientaciones estéticas tan aparentemente lejanas aunque aunadas en lo que se refiere a su conflicto esencial.

\section{Los transi como precedente artístico en torno a la dualidad espíritu y cuerpo}

La doble manifestación de lo sagrado y lo profano representará, qué duda cabe, el conflicto entre un arte para la vida y un arte para la muerte, un arte idealizador o un arte apegado a la materialidad de los cuerpos donde no habrá cabida para el dominio del espíritu como voluntad de vida. ${ }^{6}$ En este sentido, resulta destacable la exaltación de la precariedad corporal manifestada durante el marco temporal en el que se desarrollarán los transi, así como la valoración de este mismo aspecto tal y como será expuesto desde la obra de Medardo Rosso.

En este orden y en lo que respecta a los transi, es conveniente destacar que no fue sino en 1396 cuando se autorizaron las primeras disecciones humanas, hecho que vendría acompañado de la acuciante necesidad de retratar anatómicamente, desde su cruda exposición física, la naturaleza del individuo. Este hecho marcharía al compás del no lejano advenimiento, poco más de un siglo después, de los primeros tratados de anatomía moderna, siendo el primero De humani corporis fabrica, publicado en 1543 por la mano de Andreas Vesalius. Estos factores surgirán al tiempo que acontecerá el advenimiento de una estética de lo macabro observada en multitud de trabajos escultóricos funerarios entre los que puede mencionarse el Sepulcro de Jean de la Grange, Cardenal de Avignon, modelo claramente denotativo de las nuevas concepciones en le medida en que mostrará en el mismo conjunto una confianza en la naturaleza infinita y eterna del espíritu, al tiempo que una comprensión del cuerpo como fenómeno pasajero, fugitivo, llamado a perderse en el seno de los tiempos. Evidentemente, este entendimiento de la naturaleza corporal, llegará propiciado, al margen de cuestiones sociales y periodos de enfermedad, por concepciones político-teológicas entre las que podemos destacar el asentamiento definitivo de la noción del purgatorio -hacia el siglo XIII- o la influencia de las nuevas órdenes monacales, ámbito en el que no puede dejarse de lado la doctrina promulgada por la reforma cisterciense. En cualquier caso, valga comentar que esencial resulta en esta cuestión seguir la pista a la idea de la Encarnación si es que deseamos tener una noción clara de los canales que

6 En relación con esta dualidad cuerpo-espíritu, Cuillot de Suduiraut mencionará: "The evolution of funerary sculpture also led to the representation of the emaciated or whitered corpse (transi), which reproduces the grim visions of the plague and recalls the vanity of the things of this world". Páginas más adelante continuará, "Late Gothic sculpture intimately mingles the sacred and profane. It expresses in turn a deep piety and an ungoverned fantasy: the taste for life and the obsessive fear of death" (CUILLOT DE SUDUIRAUT, Sophie, "Glow and afterglow of Gothic (1400-1530)", en DUBY, G. y J-L. DAVEL (eds). Sculpture from Antiquity to the Present Day: From the Eight Century BC to the Twentieth Century. Colonia/ Londres: Taschen, 2002, 471-544, pp. 484 y 505). 
irá tomando en occidente ya desde los primeros siglos del cristianismo esta dualidad entre cuerpo y espíritu. ${ }^{7}$

Por otra parte, y retomando el simbolismo enorme ofrecido por los transi y su desarrollo a lo largo de varios siglos, no podemos ver en ellos sino un modelo estético perfectamente indicativo no sólo de la espiritualidad de una época, sino también del punto de partida de esa orientación espiritual así como del modelo de evolución a seguir a partir de ese detonante de los tiempos modernos que será la Reforma protestante. ${ }^{8}$ No entraremos en este amplio y complejo tema pero nos quedaremos, por significativo, con la clara separación acaecida en esta época entre forma inalterable y materia perecedera.

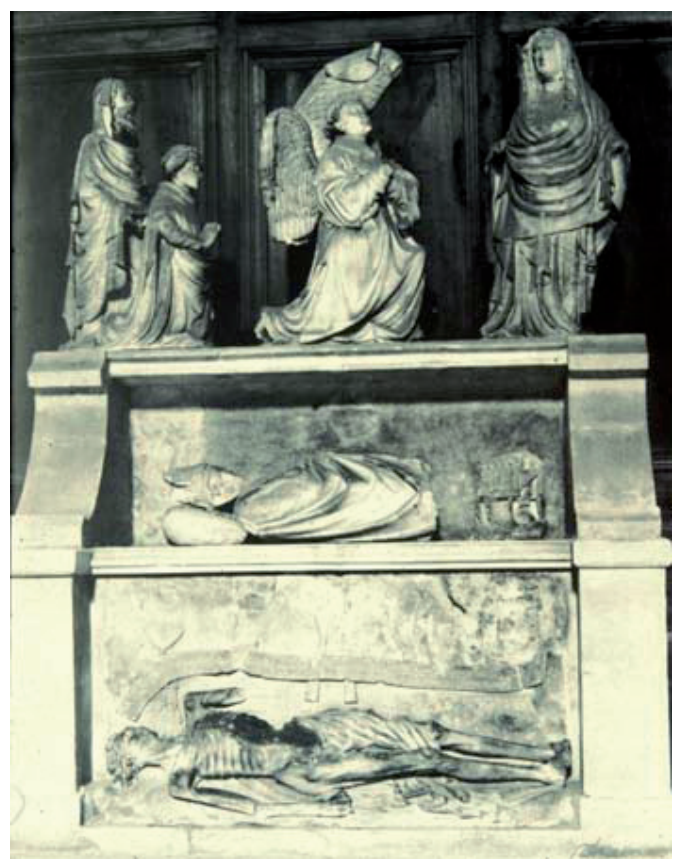

Fig. 3. Sepulcro de Jean de la Grange de la Catedral de Avignon, 1402.

\footnotetext{
7 Una aproximación general a la cuestión la podemos encontrar perfectamente estructurada en la obra de Hans Küng, El Cristianismo.

8 El desarrollo de la alteridad espíritu-materia en esta época de transición de la Edad Media a la Moderna, con atención incluida al motivo de los transi, ha sido estudiado de forma insuperable por Ernst H. Kantorowicz en su trabajo Los dos cuerpos del rey. De la presente obra citamos las siguientes líneas por denotativas de un clima de retroalimentación entre el arte, la teología y la política de una época: "Quizá nunca, salvo en aquellos siglos del 'gótico tardío', fue la mentalidad occidental tan plenamente consciente de la discrepancia entre la transitoriedad de la carne y el esplendor inmortal de una dignidad que la carne supuestamente representaba. [...] No debemos olvidar que la misteriosa yuxtaposición de un cadáver putrefacto y una dignidad inmortal tal como se exhibían en los monumentos sepulcrales, o la marcada dicotomía de la lúgubre comitiva funeraria que rodeaba al cadáver y la carroza triunfal de una efigie-momia cargada de insignias [...], se desarrollaron en el mimo clima intelectual en el que las doctrinas relativas a los 'dos cuerpos del rey' alcanzaron su formulación definitiva" (KANTOROWICZ, Ernst H., Los dos cuerpos del rey. Un estudio de teología política medieval. Madrid, Akal, 2012, pp. 426-427).
} 


\section{Lo difuso, camino hacia lo abstracto}

Con esta breve comentario acerca de la implicación de los transi en el asunto que nos ocupa se ha pretendido destacar un concepto recurrente y definitorio de la escultura de Rosso, a saber, la comprensión de la materia desde su corrupción y, por consiguiente, desde su fugacidad, desde su fragilidad, lo que llevará al artista a realizar una serie de figuras a modo de espectros en tránsito hacia lo amorfo o mortecino, cuyos contornos van a quedar desdibujados hasta no dejar sino una leve reminiscencia de aquello que ha quedado sepultado. Señala Vanesa Nicolson al respecto que: "the quality of apparition Medardo Rosso strove for in his sculpture given his figures a ghost-like power", ${ }^{9}$ una apariencia fantasmal observada de modo nítido, por ejemplo, en su Madame X, comprendida como derrota de la materialidad del ser al no quedar del rostro sino una forma "where shadows advance and bodies sink into the terrestrian plane". ${ }^{10}$ Lo espectral de la representación se descubrirá en todo momento como enterramiento del individuo en el seno de una naturaleza impasible ante cualquier deseo de individualidad o de trascendencia.

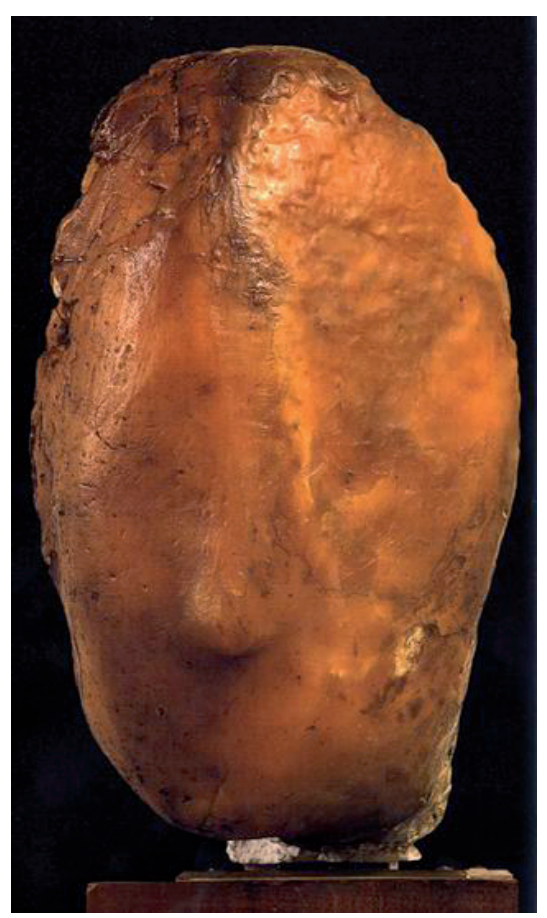

Fig. 4. Medardo Rosso, Madame X, 1869-1913. Galleria Internazionale d'Arte Moderna, Venecia.

9 NICOLSON, Vanessa, “Rosso's Drawings”, en CARAMEL, L. (com.), Medardo Rosso. Londres: South Bank Centre, 1994, 45-57, p. 45.

10 MOLA, Paola, op. cit., p. 24. 
Cuanto presenciemos se mostrará como estética de lo difuso y progresivamente desindividualizado, como consumación de la derrota del hombre frente a una naturaleza que acaba por engullirle dejando de él tan sólo unas formas entregadas a lo larvario, a lo carente de energía. La obra de Rosso, en consecuencia, podrá ser comprendida como firme avance desde una concepción idealista de la naturaleza hacia un materialismo expositivo del desarrollo de unos tiempos velados a todo entendimiento de la vida desde su riqueza espiritual. La materialidad de los seres, lejos de observarse como plena presencia bajo el haz de los rayos solares, se descubrirá entregada a las dentelladas del tiempo, quedando un halo de tristeza y nostalgia ante la impavidez mostrada por el continuo desarrollo metamórfico de la materia. Un tinte existencialista, por consiguiente, será presentido a través de unas obras que van a situar al ser frente a la nada mostrando sus contornos como prestos a deshacerse, a desfigurarse en el cuerpo de una cera elegida como material privilegiado por Rosso para la elaboración de buena parte de sus trabajos.

Lo vaporoso, lo irreal, se erigirá como presupuesto de una estética que dominará, desde la vaguedad, el desarrollo de la escultura moderna: "His images are almost hallucination that are either translated by hand directly into wax [...] These are spaces described through super-impositions and collages in which the inside and outside exist simoultaneously, the above and below appear together". ${ }^{11}$ Esta técnica tendente a la representación de un orden dual de elementos arrastrados en una misma dirección, encontrará sus fuentes próximas en la llamada Scapigliatura milanesa propia del segundo tercio del XIX. Cabe recordar que este movimiento expandido al conjunto de las artes, nacerá como respuesta a la recreación permanente de formas deudoras de la tradición clásica, de modo que, frente a una concepción unitaria de la obra, se defenderá una estética llamada a resaltar el abismo existente entre realidad e ideal, motivo que, en el ámbito de las artes plásticas, vendrá acompañado de un gusto por reflejar las desavenencias entre materia y espíritu, tal y como resulta posible observar a través de la representación de la muerte, la decadencia física o la enfermedad. Testimonios de esta corriente los poseemos en la obra pictórica de Tranquilo Cremona, Daniele Ranzoni, o en la escultura de Giuseppe Grandi, donde podemos apreciar una notable tendencia hacia lo informe, tal y como observamos en su magnífica Pleureuse, próxima en no pocos detalles al Balzac de Rodin, ventana abierta hacia la estética moderna.

Con este legado rupturista ofrecido por la Scapigliatura se encontrará en sus años de aprendizaje Medardo Rosso, cuya juventud coincide en tiempo con el apagamiento de la recién mencionada corriente estética, cuya extensión abarcó todo el norte de Italia. Así, según constata Luciano Caramel, "in the paintings of the Scapigliatura artists and the sculpture of Grandi, Rosso discovered radical techniques for blurring the outline, attenuating the edges of intersecting planes and creating a vibrant light and atmosphere, which all contributed to the overall unity of the image". ${ }^{12}$ Esta técnica enfocada a la presentación de lo borroso, de lo difuso, quedará asumida por Rosso

11 MOLA, Paola, op. cit., p. 25.

12 CARAMEL, Luciano, "The Sculptural Revolution of Medardo Rosso", en CARAMEL, L. (comp.). Medardo Rosso, Londres, South Bank Centre, 1994, 9-43, p. 12. 
desde sus inicios dado que fue en el temprano año de 1883 cuando comenzó a realizar sus primeros modelos en cera, material que emplearía con insistencia a lo largo de toda su trayectoria creativa. En relación con este punto concerniente a la técnica empleada, Sharon Hecker señalará que "Rosso used a rapid process of modeling based on a swift visual 'impression' transferred as directly as possible from his eye through his hands to the clay". ${ }^{13} \mathrm{La}$ idea de la fugacidad de lo vital quedará expresada, según vemos, mediante el difuminado de aquellos factores que dotan de contorno a la forma. Hecker continuará hablando de un proceso de desmaterialización de la obra como punto de partida de la estética contemporánea. Es posible descifrar, en todo ello, el ya mencionado deseo de superación de un canon estereotipado de belleza, y su consiguiente sustitución por un arte que venía a manifestar una cosmovisión alternativa frente a aquellas nociones aún perdurables en occidente cada vez con menos eficacia. ${ }^{14}$

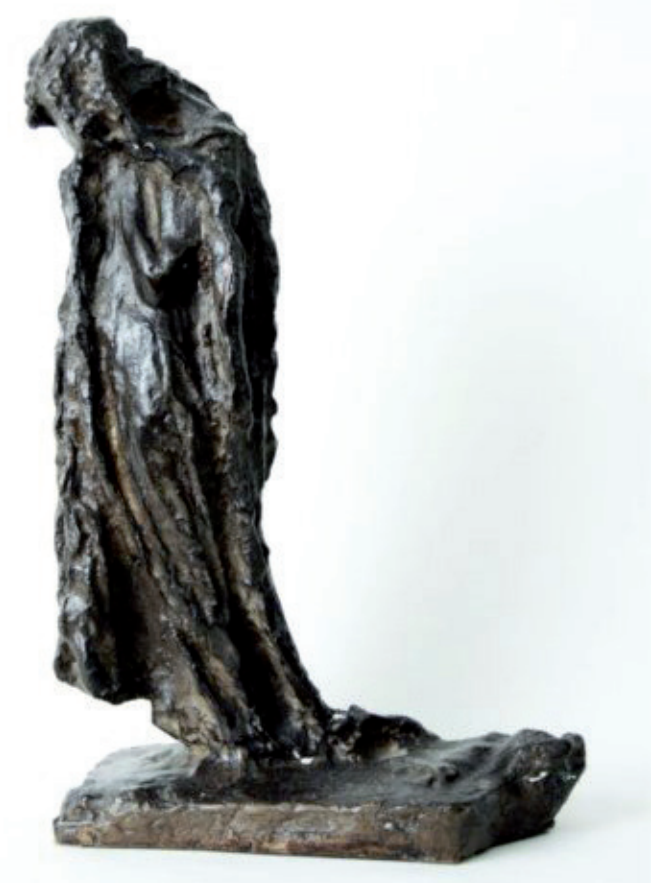

Fig. 5. Giuseppe Grandi, La Pleureuse, 1875-1878. Colección privada.

13 HECKER, Sharon, "Reflections on Repetition in Rosso's Art". En: COOPER, H. y SHARON HECKER (eds.). Medardo Rosso. Second Impressions. New Haven / Londres: Yale University Press, 2003, 34-37, p. 31.

14 En lo referente a aquellos aspectos que distinguen a Rosso como precursor de la escultura moderna, cabe mencionar las consideraciones realizadas al respecto nuevamente por Sharon Hecker. Según éste: "Such a view of the solid, three-dimensional object as subject to the influence of time and its surrounding atmosphere is considered Rosso's most important contribution to modern sculpture. His attempts to dematerialize sculpture greatly interested Rodin, Umberto Boccioni, André Mason, Alberto Giacometti, and Henry Moore. [...] Rosso cultivated this paradoxical situation by calling his sculptures «impressions» in both Italy and France without specifyng the source or exact meaning of the term". (HECKER, Sharon, op. cit., p. 27). 
El aludido concepto de desmaterialización, entendido como pérdida de densidad material de la figura, se propondrá como primer paso dado desde un arte representativo hacia uno abstracto, lo que desde un plano paralelo se comprenderá como medio adecuado de cara a la intelección del fenómeno estético no desde la apariencia del ser, sino desde una escondida profundidad que, en función de qué creador provenga, podrá ser presentida ya como espacio divino, ya como oscuro reino de la nada. Esto último es lo que observaremos en la obra de Rosso como sintomático de unos tiempos entregados a la idea de decaimiento y caducidad. La crisis de esta alteridad espíritumateria será planteada en un primer momento como pérdida de consistencia del primer elemento; sólo más adelante, una vez se ha realizado la tabula rasa de aquellos conceptos adheridos a ambos términos, esta misma alteridad podrá ser tratada como incipiente espiritualización, precisamente, de lo que hasta hace poco comprendíamos como mera existencia material.

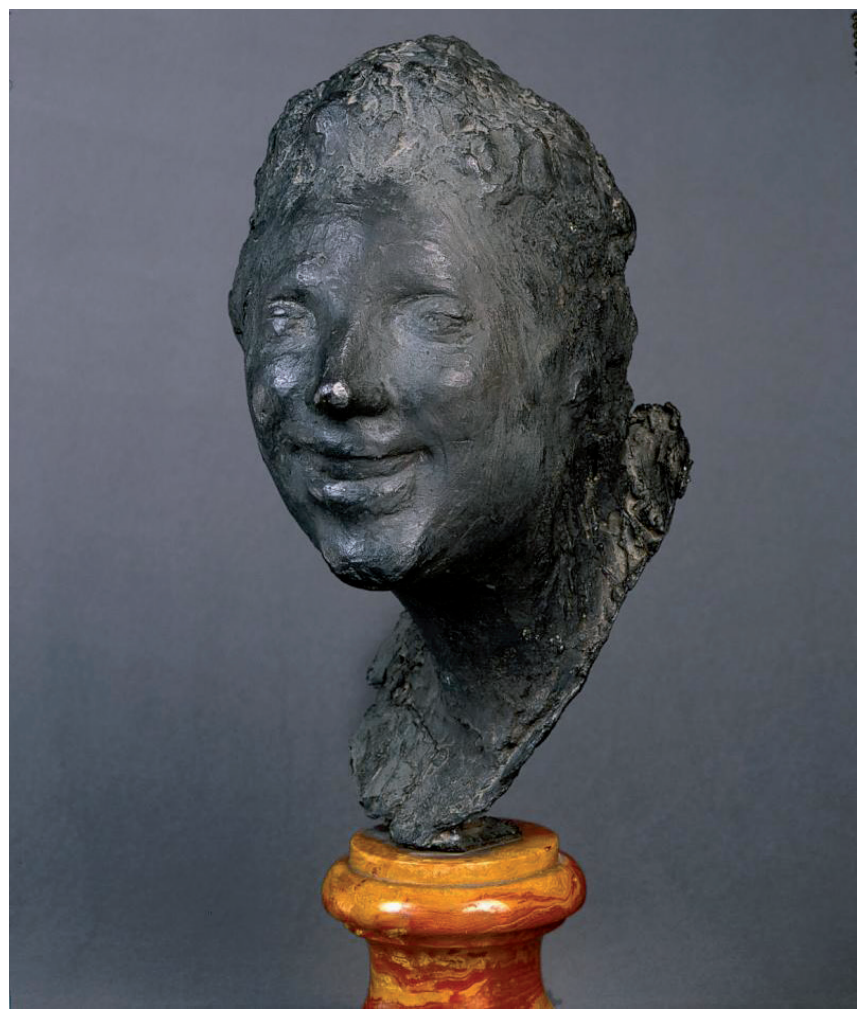

Fig. 6. Medardo Rosso, Rieuse, 1890. Musée Rodin, Paris.

Será entre estos límites donde quedará recluida la escultura del artista italiano, permaneciendo el cuerpo de la obra como espacio plurivalente en la medida en que el modelo va a quedar despojado de contrastes, de tensiones bruscamente enfrentadas, provocando así la aparición de una superficie fantasmal, silenciosa, que observada 
desde aquella única perspectiva cuya luz habrá de perderse al momento, revelará fulminantemente un solo aspecto, un solo matiz capaz de mostrar una verdad más poderosa que todo el expresivo mutismo descifrado a lo largo del recorrido completo realizado alrededor del objeto escultórico. Esa verdad, ese destello luminoso, esconderá en su seno el encuentro del ser con la inmensidad de lo eterno, como queriendo ocultar más que desvelar el punto, el instante exacto, donde el fenómeno se hace uno con lo numinoso, más allá del carácter trascendental o nihilista que a aquél le otorguemos.

Una vez más, será Hecker quien describa este hecho al afirmar que "Rosso suggests the precarious vision of an idea momentarily caught in the process of forming and dissolving". ${ }^{15}$ En este último, "the figure is so blurry that it seems about to dissolve into a equally nebulous background". ${ }^{16}$ Será precisamente la idea súbita citada por el autor la que exprese con su simple trazo aquello que calla el resto de la composición, revelando mediante un fenómeno intuitivo cuanto escapa al análisis escaso de lo que el resto de la composición nos habrá ofrecido. A través de su escultura, por consiguiente, Rosso propondrá una estética del detenimiento, de lo contemplativo y lo sugestivo más que de lo crítico, logrando romper la alternancia entre obra y espectador para desvelar un hecho no definible como representativo ni como abstracto, sino como absoluto, verdadero y único, en la medida en que su correcta comprensión escapará a un ámbito puramente fenoménico.

\section{Consideraciones últimas sobre la obra y la pervivencia de Medardo Rosso}

Cuanto se ha querido poner de relieve con la realización de este trabajo es la presentación de Medardo Rosso como iniciador de una escultura moderna y rupturista con los modelos heredados del pasado. A través de estas páginas hemos podido observar los transi como fenómeno estético donde se va a reflejar de modo explícito la separación entre cuerpo y espíritu emprendida ya en la escultura europea de finales del medievo. A través de las macabras formas presentadas por estas lejanas composiciones funerarias hemos podido observar el retrato del cuerpo como objeto caduco entregado a la putrefacción, al tiempo que un espíritu expuesto como contrapeso victorioso de esa misma naturaleza carnal. Este hecho, deudor de un cisma en la religiosidad de la época, comprenderá la individualidad como marco donde fuerzas espirituales y fuerzas materiales van a quedar enfrentadas, quedando abierta así la brecha que, con el tiempo, llevará a una separación radical, cuando no a la supresión, de la unidad alma-cuerpo.

Por otro lado, hemos podido comprobar cómo una visión dionisiaca de la vida, no racionalizada, se desvelará a través de la obra de arte a modo de ruptura respecto de los postulados defendidos con firmeza hasta bien entrado el siglo XIX. Dentro de este marco renovador hemos observado el nacimiento de la Scapigliatura o «desmelenamiento» acaecido en el norte de Italia durante la segunda mitad del XIX, corriente

15 HECKER, Sharon, op. cit., p. 45.

16 HECKER, Sharon, op. cit., p. 46. 
estética de fuerte impronta en un Rosso que, nacido en Turín, conviviría asiduamente con dichas formas ya desde sus albores creativos tomando como modelo principal tanto la obra pictórica de Cremona y Ranzoni, como la escultura de Grandi, caracterizadas todas ellas por los trazos difuminados así como por un resaltamiento de lo grotesco y de la dualidad marcada e irresoluble entre espíritu y materia.

Así mismo, la obra de Rosso ha sido tratada como precursora de la escultura contemporánea a modo de inicial punto de fuga hacia una organicidad y un dinamismo más y más acentuado con el paso de los años, llegando hasta nuestros días como detonante de la idea a expresar por no pocos creadores -Tony Cragg por citar uno de entre los más destacados-, cuyas figuras entre lo orgánico y lo inorgánico, entre lo representativo y lo abstracto, mostrarán un gusto especial por desvelar el proceso en curso, la fuerza dinámica que alienta la obra de arte.

Por todo ello, como iniciador de una estética hoy viva y revalorizada, Medardo Rosso se presenta como enclave central de cara a observar aquellos detalles, aquellos trazos que, a modo de gozne, manifestaron el decaimiento de la escultura clásica al tiempo que abrieron espacios hasta el momento ensombrecidos, espacios por donde habrían de adentrarse de lleno los primeros exponentes de una modernidad ya planteada desde las vanguardias y, tras ellas, un sinnúmero de artistas obcecados en acercarse a la obra de arte desde su comprensión como fenómeno vivo, como exponente de unos tiempos donde el individuo completo, como organismo espiritual, ha sustituido la activa y honda percepción por un escaso y superficial empleo de los sentidos. 